\title{
Design of Voltage Regulating Control Device of Improved PID Algorithm for the Vehicle AC Generator Based on DSP
}

\author{
Du Baojiang ${ }^{1}$, Guo Jingmin ${ }^{1}$, Ji Changqin ${ }^{1}$, Wei Xiong ${ }^{1} \&$ Sun Anbo $^{1}$ \\ ${ }^{1}$ College of Mechanical Engineering, University of Shanghai for Science and Technology, Shanghai, China \\ Correspondence: Guo Jingmin, College of Mechanical Engineering, University of Shanghai for Science and \\ Technology, 516 Jun Gong Road, Shanghai 200093, China. Tel: 86-188-0192-8767. E-mail: \\ gjmshanghai@126.com
}

Received: March 5, 2012

Accepted: March 26, 2012 Online Published: May 11, 2012

doi:10.5539/mas.v6n6p7

URL: http://dx.doi.org/10.5539/mas.v6n6p7

The project of science and technology commission of Shanghai municipality: Study of virtual manufacturing technology and service model for equipments (11DZ1121300)

\begin{abstract}
The performance of the voltage regulating control system will have a direct impact on the power supply quality of vehicle generator. Therefore this paper researches a design of voltage regulating control device of improved PID algorithm for the vehicle alternating current (AC) generator based on DSP. According to the analysis of the operation and the voltage regulating principle of the vehicle AC generator, using the TMS320LF240 DSP as controlling chip, the optimized AD signals conditioning circuit, PWM circuit and improved increment PID algorithm excitation controller based on digital PID algorithm are designed, which only use integral controller by the incremental algorithm, and the proportional and derivative controller by the positional algorithm. After integrated debugging and simulation, the reliability and high precision of the voltage control system are validated.
\end{abstract}

Keywords: vehicle, AC generator, voltage regulating, DSP, signals conditioning, increment PID

\section{Introduction}

The vehicle electrical equipment needs a stable supply voltage. But vehicle generator has a big range of revolving speed, so the voltage regulating control device is very important to guarantee the quality of generator power supply. The performance of the voltage regulating control system will have a direct impact on the power supply quality of vehicle generator. The analog excitation voltage regulator has problems, for example serious drift with temperature changing, inconvenient maintenance and low precision.

This paper presents a new type of voltage generator excitation control system based on TMS320LF240 DSP which has powerful data processing ability, high-performance static CMOS technology and high-speed real-time control ability. Using the TMS320LF240 DSP as controlling chip, the optimized AD signals conditioning circuit, PWM circuit and improved increment PID algorithm excitation controller based on digital PID algorithm are designed.

\section{The System Design}

\subsection{Working Principle}

With the engine running, the generator output voltage $\mathrm{U}$ can be is calculated as follows.

$$
\mathrm{U}=\mathrm{C} * \mathrm{~B} * \mathrm{n}-\mathrm{I} * \mathrm{R}
$$

C ---- Structure constant of the generator;

I ---- Generator output current;

B ---- Generator excitation magnetic flux;

$\mathrm{n}$---- Generator speed;

$\mathrm{R}$---- Armature winding resistance of the generator. 
It can be concluded from above that the output voltage $U$ of the generator changes with the generator speed $n$ and the load IR. To keep the stability of the output voltage generator, the field winding current intensity or direction and the regulate air gap of the magnetic field can be adjusted.

\subsection{The Overall Design}

Using TMS320LF240 DSP as the control chip, a kind of optimized AD conditioning circuit and an improved incremental PID algorithm are developed, and the output voltage of the vehicle AC generator is regulated by the PWM circuit. First of all, the output voltage has to be detected, as figure1, then the generator three-phase AC voltage is changed into DC voltage by AD signal conditioning circuit, and converted to digital signal by AD converting circuit, then put into the input of the DSP, DSP regulates the field current intensity or direction by PWM circuit after the improved incremental PID algorithm processing, at last, the generator's excitation is controlled through the drinving circuit to have the generator output a stable voltage. So the device must have Software of DSP and the auxiliary circuit including analog signal conditioning, AD convertion, PWM, driving and congtoll circuit. Figure 1 shows the overall scheme of the system design.

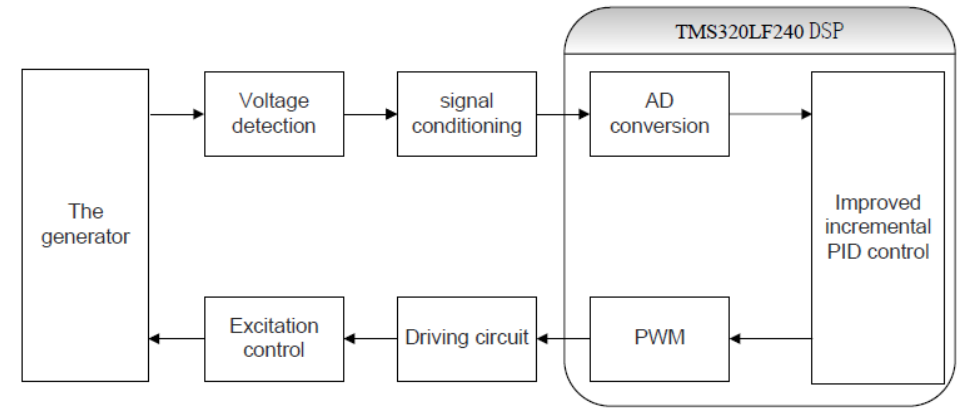

Figure 1. The overall scheme of the system design

\section{DSP Software Design}

\subsection{The Overall Software Design}

Generator voltage control is a fast real-time closed-loop control system, and the regulating activities have to be quickly realized on the quikly changing of the voltage, so the software system may include the system and the clock configuration module, AD conversion module, filter module, PID control algorithm module, and PWM output module, to process the real-time regulating signals. The Figure 2 shows the overall software diagram.

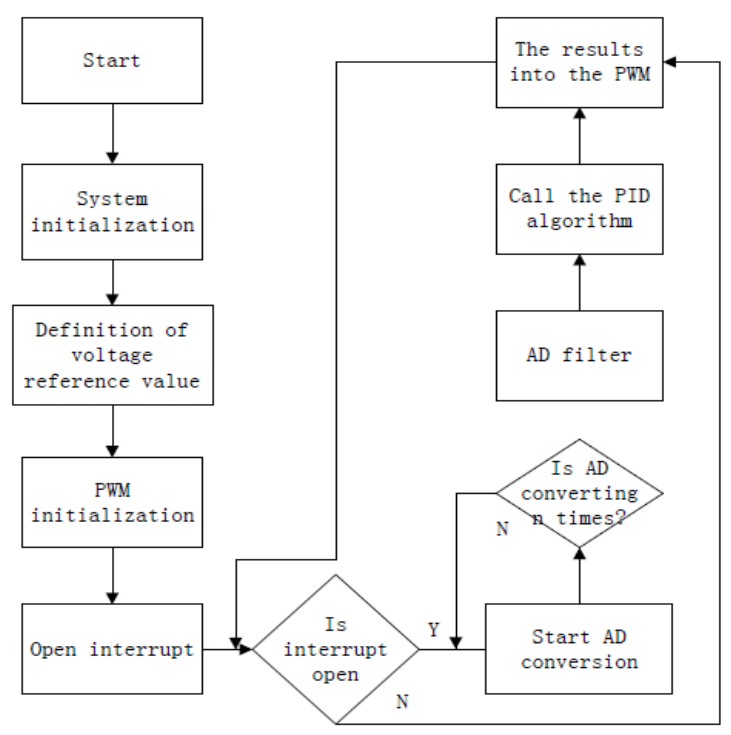

Figure 2. The overall software design 


\subsection{Improved Increment Type PID Excitation Control}

The generator terminal voltage deviation works as PID algorithm input, after PID algorithm processing, results are sent into PWM controller. The quality of PID excitation control directly decides the quality of the voltage regulation system. Simply put, the correction function of PID excitation controller is as follows: the proportional part rapidly proportionally reflects the generator terminal voltage deviation signal, and once deviations formed, the controller immediately generates a control role, in order to reduce the deviation, maintain the generator voltage constant. The integral part is mainly to eliminate the steady-state error of excitation system, improve the system of indiscrimination degree. The differential part reflect the trend of the deviation signal changing, and before the error signal becomes too large, an effective correction signal is early introduced to the system, thus the operation speed of the system is speeded up, the adjusting time is reduced.

Compared with Positional PID algorithm, incremental PID algorithm requires deviation parameters of the first two moments. The integrating part of positional PID algorithm needs the large amount of calculation, and proportional and differential calculation amount is small. According to the advantages and disadvantages of the two algorithms, in this article only integral part is calculated by incremental operation, the other two are calculated by positional operation, developed a PID algorithm combining positional algorithm with incremental algorithm. The proportion, integral, differential controller can be independently added, therefore, PID excitation controller only use integral controller by the incremental algorithm, and the proportional and derivative controller by the positional algorithm. The algorithm represent as follows:

$$
\begin{gathered}
\mathrm{U}(\mathrm{k})=\mathrm{UP}(\mathrm{k})+\mathrm{UI}(\mathrm{k})+\mathrm{UD}(\mathrm{k}) \\
\mathrm{UP}(\mathrm{k})=\mathrm{KP} * \mathrm{e}(\mathrm{k}) \\
\mathrm{UI}(\mathrm{k})=\mathrm{UI}(\mathrm{k}-1)+\mathrm{KI} * \mathrm{e}(\mathrm{k}) \\
\mathrm{UD}(\mathrm{k})=\mathrm{KD}^{*}[\mathrm{e}(\mathrm{k})-\mathrm{e}(\mathrm{k}-1)]
\end{gathered}
$$

$\mathrm{k}$---- Sampling serial Numbers, $\mathrm{k}=0,1,2 \ldots$;

$\mathrm{U}(\mathrm{k})$---- Output value of the PID controller in the $\mathrm{k}$ time sampling time;

$\mathrm{e}(\mathrm{k})$---- Deviation value in the $\mathrm{k}$ time sampling time;

$E(k-1)$---- Deviation value in the (k-1) time sampling time;

$\mathrm{KP}$---- Amplification factor;

KI ---- Integral coefficient;

KD ---- Differential coefficient.

\section{The Circuit}

\subsection{Signal Conditioning Circuit}

In order to achieve accurate and effective control, signal conditioning must be carried out before sensors signals come into AD converter. Signal conditioning concludes resistor dividing the voltage, differential amplifying and filtering. TMS320LF240 DSP contains a single polarity ADC with 16 analog channels, the minimum conversion time is $375 \mathrm{~ns}$, and 16 channels can be arranged in any order and converted automatically, conversion results are sequentially placed. Therefore, this design uses the ADC of DSP.

The range of the analog input voltage of the ADC is from 0 to $3.3 \mathrm{~V}$, but the generator output voltage is about 14 $\mathrm{V}$. So the generator output voltage signal must be processed fist by stepping down to 0-3.3 V. Figure 3 shows the AD signals conditioning circuit.

The generator output voltage VOUT stepped down by resistance R1 and R2, so the function between the voltage $\mathrm{U} 1$ and the voltage $\mathrm{U}$ is $\mathrm{U} 1=\mathrm{R} 1 /(\mathrm{R} 1+\mathrm{R} 2) \mathrm{U}$, the voltage $\mathrm{U} 2$ by the benchmark TL431 defined is $\mathrm{U} 2=2.5(1$ $+\mathrm{R} 4$ / R5). The output voltage U3 after the input voltage U1 and U2 difference amplifying is U3 = R9 / (R8 + R9) * $(1+\mathrm{R} 7$ / R6) U1-(R7 / R6) * U1, make R4 = R5 = R8 = R9, the above function simplified to U3 = U1-U2. Let $\mathrm{R} 1=20 \mathrm{~K} \Omega, \mathrm{R} 2=10 \mathrm{~K} \Omega, \mathrm{R} 4=\mathrm{R} 5=0 \Omega ; \mathrm{R} 6=\mathrm{R} 7=\mathrm{R} 8=\mathrm{R} 9=1 \mathrm{~K} \Omega, \mathrm{U} 2=2.5 \mathrm{~V}$, so the relationship between the voltage $\mathrm{U} 3$ with the voltage $\mathrm{U}$ is $\mathrm{U} 3=\mathrm{U} / 3-2.5$. Therefore, if the voltage of the generator voltage $\mathrm{U}$ was between 7.5 and 16.5, and the ADC input signal would be from 0 to $3.3 \mathrm{~V}$; If $\mathrm{U}<7.5 \mathrm{~V}$, the amplitude limit the ADC input signal in $0 \mathrm{~V}$; If $\mathrm{U}>16.5 \mathrm{~V}$, the amplitude diode limit the ADC input signal in $3.3 \mathrm{~V}$. It can prevent DSP over-voltage and burned, and help to optimize the generator voltage control device also. 


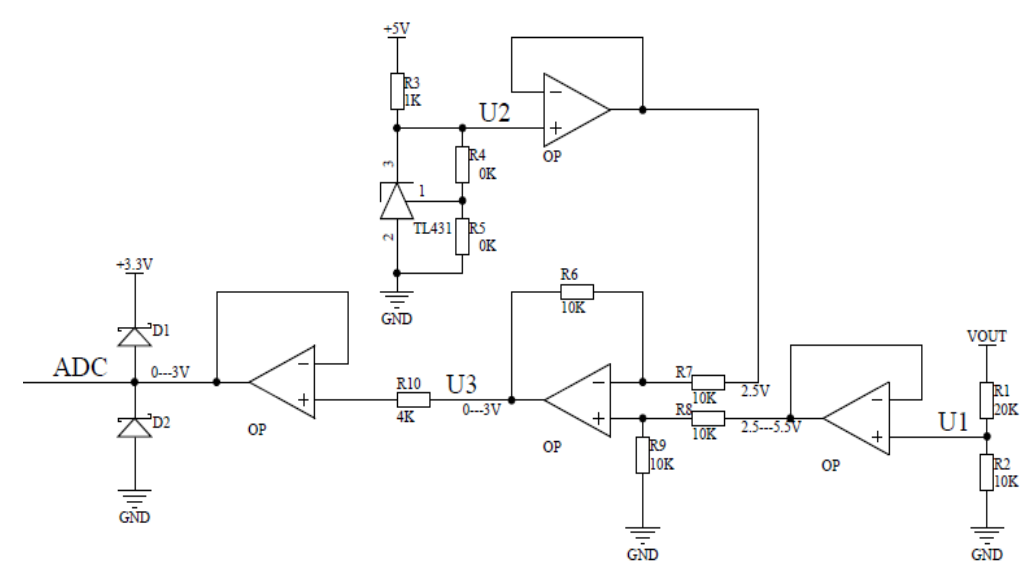

Figure 3. The AD signal conditioning circuit

\subsection{PWM Circuit}

In the PWM technology, power devices work in switch state, to change the average load voltage, through changing the opening and cut-off time of the driving pulse signal of the power components. This is also the basic principle of PWM regulating the speed and voltage. The Figure 4 shows the PWM circuit.

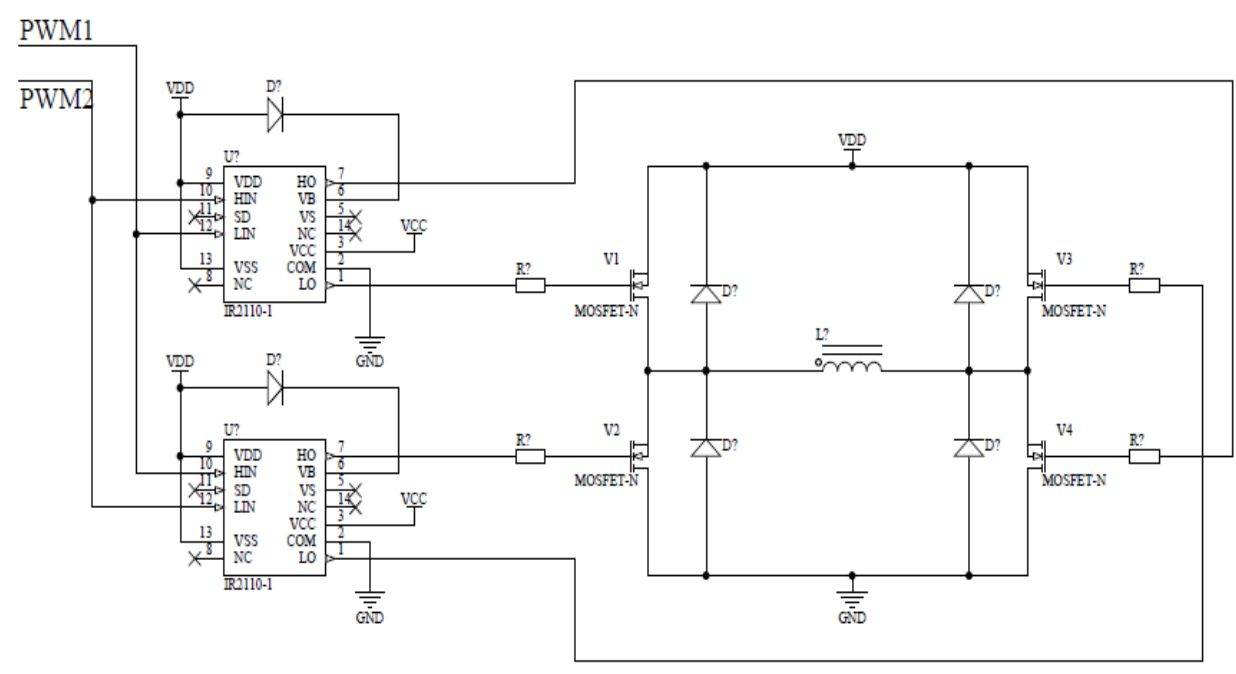

Figure 4. The PWM circuit

\subsubsection{PWM Output Driving Circuit}

Driving circuit is interface between the main circuit and the control circuit. Using the good performance of driving circuit can make power semiconductor devices work in an ideal switch state, short the switch time and lower switch loss. In the design the output PWM signal of DSP drives H bridge power circuit by the chip IR2110. When the MOS door devices are used in the source or emitter output circuit or as a bridge arm power tube, the suspension drive circuit has to be used to drive the grid. This means the voltage should float with the change of the electric potential of source and emitter. IR2110X is special two MOSFET gate driving integrated circuit, this drive circuit can drive a high voltage side and a low voltage side. The functions of the MOSFET is integrated in a package, they work based on the bootstrap principle. When the high voltage side and low voltage side components are driven, independent drive powers are not necessary, therefore circuits are simplified and the switch speed is improved and an ideal drive waveform can be obtained.

\subsubsection{H Bridge Circuit}

This design uses bipolar reversible PWM mode, its characteristic is that in a switch cycle, the armature voltage polarity is alternating between the positive and the negative to control the intensity and the direction of the 
current by adjusting PWM duty cycle. If the duty cycle $\alpha$ is more than $50 \%$, excitation current flow forward; If the duty cycle $\alpha$ is less than $50 \%$, excitation current flow back; If the duty cycle $\alpha$ equals $50 \%$, excitation current is zero. Here the direction of excitation current is relative.

\section{Experiment}

The rated voltage generator is $14 \mathrm{~V}$. In order to check its control and the dynamic performance, the system is tested in two different conditions, the incremental PID parameters are: $\mathrm{KP}=1.8, \mathrm{KI}=0.2, \mathrm{KD}=0.35$. (1)When the generator speed is under a constant, changing the load, observe the change of the generator output voltage, such as shown in Table 1. (2)When the generator is in the low load, changing the speed from 1800 to $5000 \mathrm{r} / \mathrm{min}$, observe the change of the generator output voltage, such as shown in Table 2.

Table 1. The generator output voltage change with load current changing

\begin{tabular}{cccccccc}
\hline Load current/A & 2 & 5.4 & 9.6 & 11.2 & 16 & 20 & 25 \\
\hline $\begin{array}{c}\text { The output } \\
\text { voltage/V }\end{array}$ & 14.07 & 14.01 & 13.88 & 13.85 & 13.8 & 13.75 & 13.7 \\
\hline
\end{tabular}

Table 2. The generator output voltage change with rotating speed changing

\begin{tabular}{cccccccc}
\hline $\begin{array}{c}\text { The rotation } \\
\text { /r/min }\end{array}$ & 1824 & 2320 & 2790 & 3276 & 3782 & 4310 & 4974 \\
\hline $\begin{array}{c}\text { The output } \\
\text { voltage/V }\end{array}$ & 13.9 & 14.08 & 14.06 & 14.02 & 14.05 & 14.07 & 14.05 \\
\hline
\end{tabular}

The results show that the output voltage of the generator after regulating can be stabled within the range of $+5 \%$, and the device has good voltage stability characteristics, so as to ensure the stability of the vehicle power supply system operation.

\section{Epilogue}

Aimed at the stable valtage ouput of vehicle AC generator, optimized AD circuit, PWM circuit are designed, using the improved incremental PID algorithm, a type of voltage regulator control is developed. After testing, it has good performance.So the regulating control device can ensure the high quality voltage to vehicle power supply, and this design has a good application prospect.

\section{References}

Ding, X. F., Liu, J. L., \& Zhang, X. (2008). Controller based on DSP for hybrid excitation constant voltage synchronous generator. Micro Motor.

Shao, W., \& Ling, D. (2010). Improved incremental PID algorithm based on intelligent integral. Mechanical and Electrical Control Technology, 39, 11.

Wu, Q. W., \& Feng, Y. (2011). The Research for the Method of DSP Calculation. Electronics \& Packaging, 11(7).

Xu, X. L., Shao,T. Z., \& Zhai, T. J. (2011). Design of excitation regulator based on DSP. Foreign Electronic Measurement Technology, 30(5). 